New Approach Combi ni ng $\mathrm{Br}$ anch and Pri ce wi th Net aheur i st i cs to Sol ve Nur se Schedul ing Probl em

\begin{tabular}{|l|l|}
\hline 著者 & $\begin{array}{l}\text { I NAFUNE J unya, WATANABE Shi nya, Okuder a } \\
\text { Nasay oshi }\end{array}$ \\
\hline $\begin{array}{l}\text { j our nal or } \\
\text { publ i cat i on t i t I e }\end{array}$ & $\begin{array}{l}\text { Jour nal of Advanced Comput at i onal I nt el I i gence } \\
\text { and I nt el I i gent I nf or nat i cs }\end{array}$ \\
\hline vol une & 21 \\
\hline number & 7 \\
\hline page r ange & $1251-1261$ \\
\hline year & 2017- 11- 20 \\
\hline URL & ht t p: //hdl . handl e. net /10258/00009662 \\
\hline
\end{tabular}




\title{
New Approach Combining Branch \& Price with Metaheuristics to Solve Nurse Scheduling Problem
}

\author{
Junya Inafune, Shinya Watanabe, and Masayoshi Okudera \\ Muroran Institute of Technology, Japan \\ E-mail: 16043007@mmm.muroran-it.ac.jp \\ [Received 00/00/00; accepted 00/00/00]
}

\begin{abstract}
This paper presents a new approach combining Branch and Price (B\&P) with metaheuristics to derive various high-quality schedules as solutions to a nurse scheduling problem (nurse rostering problem). There are two main features of our approach. The first is the combination of $B \& P$ and metaheuristics, and the second is the implementation of an efficient B\&P algorithm. Through applying our approach to widely used benchmark instances, the effectiveness of our approach is determined.
\end{abstract}

Keywords: nurse scheduling problem, branch-andprice, metaheuristics, evolutionary multi-objective optimization

\section{Introduction}

The nurse scheduling problem (NSP), also called the nurse rostering problem, is a typical scheduling problem and well known as an NP-hard problem. The characteristics of NSP are that constraints are intricately entangled, making it very difficult to find a schedule with no constraint violations.

According to a report by Ikegami[1], most chief nurses spend an average of 11 hours creating a shift schedule every month.

Therefore, there is a strong need for a way to create a shift schedule automatically. There have been many approaches to NSP proposed. These approaches can be divided into two categories: the mathematical programmatic exact approach and the heuristic approach. These approaches have completely different features. The mathematics approach can derive an exact, optimal solution, but it is difficult to apply this approach to large-scale problems involving a large number of nurses, and it cannot be used to create a variety of schedules. On the other hand, the heuristic approach is good for creating various schedules at one time, and it is better than the mathematics approach for attaining good solutions to large-scale problems. However, the quality of solutions derived by the heuristic approach is not assured, and it is difficult to get an acceptable schedule in high-difficulty problem situations.

Recently, the branch and price $(\mathrm{B} \& \mathrm{P})$ has interested many researchers because of its high usefulness for many combinational problems, such as the traveling salesman problem, vehicle routing problem, and NSP. The B\&P is a combination approach of column generation plus branch and bound, and its implementation requires a certain degree of knowledge and technique. In addition, for it to be effective, ingenuities in the area of tree searching and branching should be adequately considered.

Maenhout and Vanhoucke took up an implementation of B\&P for the NSP and investigated how much influence the different settings of parameters have on the results[2]. Burke and Curtois provided an effective approach to B\&P for NSP and presented outstanding results for different benchmark instances[3].

However, like other mathematical programming methods, the B\&P approach cannot produce various types of high-quality schedules. Since it is very difficult to predefine every criterion and every constraint as a mathematical model in many actual hospitals, most head nurses want several candidate schedules with different nurse assignments. However, no existing B\&P approaches can resolve this big problem.

We therefore present a new approach that uses B\&P to derive many different, high-quality schedules within practicable computing times.

The key element of our approach is a combination of a mathematical programming method (B\&P) and a heuristic algorithm. Please note that the aim of our approach is not to solve a large-scale problem but to derive many high-quality schedules. There are two features of our approach. The first is a more efficient implementation of $\mathrm{B} \& \mathrm{P}$, and the second is a combination of $\mathrm{B} \& \mathrm{P}$ and a heuristic algorithm. In order to obtain many high-quality and high-diversity schedules, we choose the evolutionary multi-objective optimization (EMO) algorithm as the heuristic algorithm. In our approach, B\&P first derives an exact schedule, and the EMO attempts to derive many different schedules having almost the same qualities as the optimal one. An important point is that EMO uses the solution derived by B\&P as an initial solution to improve search efficiency. In this paper, we have termed our approach "B\&P-EMO".

We used open benchmark instances on the website "Employee Shift Scheduling Benchmark Data Sets" [4] to verify the effectiveness of our B\&P-EMO. This website has a collection of many different benchmark instances 


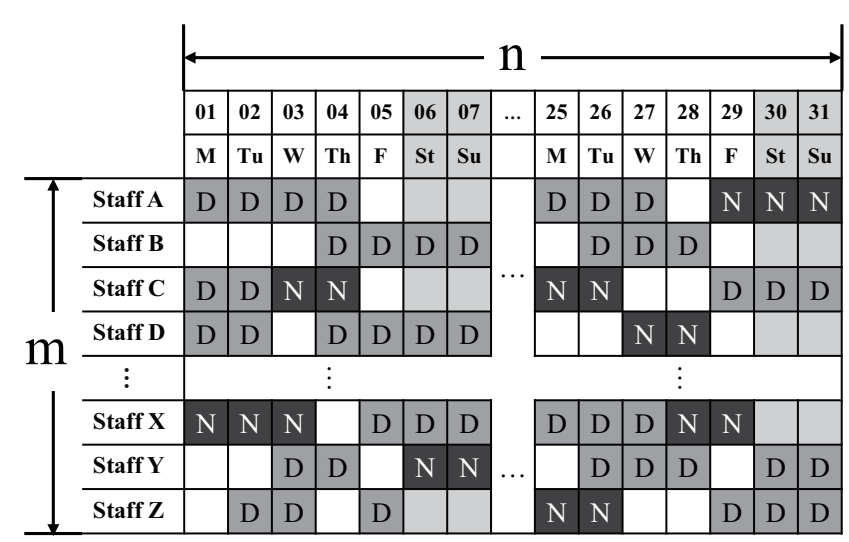

Fig. 1. Example of a nurse schedule

that have been used in related papers, and it offers these instances and the most satisfactory solutions to the public. These instances are categorized according to the size of the problems. Some typical problems on this website are listed below.

Small:

- 14 days, 11 nurses, 1 shift types (Musa[5])

- 7 days, 14 nurses, 2 shift types (Ozkarahan[6])

Medium:

- 30 days, 28 nurses, 2 shift types (Ikegami-2ShiftDATA1[7])

- 31 days, 16 nurses, 4 shift types (ORTEC01[8])

Large:

- 42 days, 51 nurses, 8 shift types (ERRVH)

- 42 days, 54 nurses, 12 shift types (MER)

In this paper, we present two different cases using medium- and small-sized instances from our experience. The first compares the results of our implementation of $\mathrm{B} \& \mathrm{P}$ with those of previous $\mathrm{B} \& \mathrm{P}$ approaches, and the second verifies the variety of solutions derived by our B\&PEMO.

This paper is organized as follows. After an explanation of the NSP in section 2, we present an outline of $\mathrm{B} \& \mathrm{P}$ in section 3 . In section 4 , we present our original approach, B\&P-EMO, which has two original features: effective implementation of B\&P and a combination of $\mathrm{B} \& \mathrm{P}$ and heuristic algorithm. Next, the results of applying B\&P-EMO to benchmark instances are given in section 5. The last section contains our conclusions.

\section{Nurse scheduling problem}

The NSP aims to create a work schedule for nurses during a specific period, such as two weeks or a month. This schedule is made up of a list of all nurses' names with their work hours, as shown in Fig. 1. In this paper, this list matrix is defined as the number of nurses $m$ and the number of periods $n(m \times n$ matrix $)$.
There are two different types of constraints in the NSP, hard and soft constraints, depending on their degree of importance. A hard constraint must definitely be satisfied, while a soft constraint does not necessarily have to. A schedule with unsatisfied hard constraints is not acceptable in a hospital setting. On the other hand, schedules with unsatisfied soft constraints can be used, but those with fewer violations of soft constraints are preferable. Therefore, a top goal of the NSP is to find a solution that satisfies every hard constraint.

The following are some of typical NSP constraints.

- Constraints in lengthwise direction (related to the quality of medical services)

- The number of nurses required per working term

- The number of groups

- Constraints in widthwise direction (related to the quality of a nurse's life)

- The number of working days for each nurse

- Regular working pattern

The above constraints can be divided into two categories: lengthwise constraints and widthwise constraints. Most of the lengthwise constraints are related to the quality of medical services; most of the widthwise constraints are related to the quality of the nurses' lives.

\subsection{Formulation of NSP}

In most cases of NSP's application, the schedule of a specific period is treated as optimization parameters and the objective of NSP is set to minimize the amount of constraint violations.

The following formula is used in both the B\&P and $\mathrm{EMO}^{1}$. Equations (1) to (5) represent parameters related to the nurse schedule. The nurse schedule is defined as the number of nurses $m$ and the number of periods $n(m \times n$ matrix $\left.\mathbf{X}_{m \times n}\right)$. Each element $x_{p q}$ of the matrix $\mathbf{X}_{m \times n}$ means a work shift $w_{r(r=1 \ldots s)}$, such as day shift or night shift, on date $q$ for nurse $p$.

We considered all constraints as either lengthwise or widthwise constraints. (6) presents the formulation of the objective function as the sum of constraint violations in the case of dividing constraints into two parts: $f^{\text {service }}(X)$ and $f^{\text {people }}(X)$. The equations in (6) correspond to (7) and (8).

$$
\begin{aligned}
\underset{m \times n}{\mathbf{X}} & =\left(\begin{array}{cccc}
x_{11} & x_{12} & \cdots & x_{1 n} \\
\vdots & \vdots & \ddots & \vdots \\
x_{p 1} & x_{p 2} & \cdots & x_{p n} \\
\vdots & \vdots & \ddots & \vdots \\
x_{m 1} & x_{m 2} & \cdots & x_{m n}
\end{array}\right) \\
x_{p q} & =w_{r} \quad\left(p \in M, q \in N, w_{r} \in W\right) \\
M & =\{1,2, \cdots, m\} \\
N & =\{1,2, \cdots, n\} \\
W & =\left\{w_{1}, w_{2}, \cdots, w_{s}\right\}
\end{aligned}
$$

1. In EMO, (6) is used as the first objective and diversity of population is used as the second objective (See section 4.3.3). 


$$
\begin{aligned}
& \text { Minimize } f(X)=f^{\text {service }}(X)+f^{\text {people }}(X) \quad \text {. } \\
& f^{\text {service }}(X)=\sum_{i=1}^{k} \omega_{i}^{\text {service }} c_{i}^{\text {service }}(X) \quad . \quad . \quad . \\
& f^{\text {people }}(X)=\sum_{i=1}^{l} \omega_{i}^{\text {people }} c_{i}^{\text {people }}(X)
\end{aligned}
$$

Each constraint has own weight based on its level of priority-a constraint with higher priority is given a heavier weighting. The meaning of each symbol in the above equations is as follows. $k$ is the number of lengthwise constraints. $l$ is the number of widthwise constraints. $c_{i}$ is the number of violations of constraint $i . \omega_{i}$ is the weight of constraint $i$.

\section{Branch and price on NSP}

Many exact mathematical techniques, such as the branch and bound method, have been put forward for the NSP. Among them, the Branch and Price (B\&P) has been proven to be effective by Maenhout[2] and E.K Burke[3]. $\mathrm{B} \& \mathrm{P}$ is a methodology that can derive an exact solution by combining column generation and branch and bound (B\&B), and it has been widely applied to assignment problems. In this section, we present the general flow of $\mathrm{B} \& \mathrm{P}$ algorithm and procedures of column generation and $\mathrm{B} \& \mathrm{~B}$.

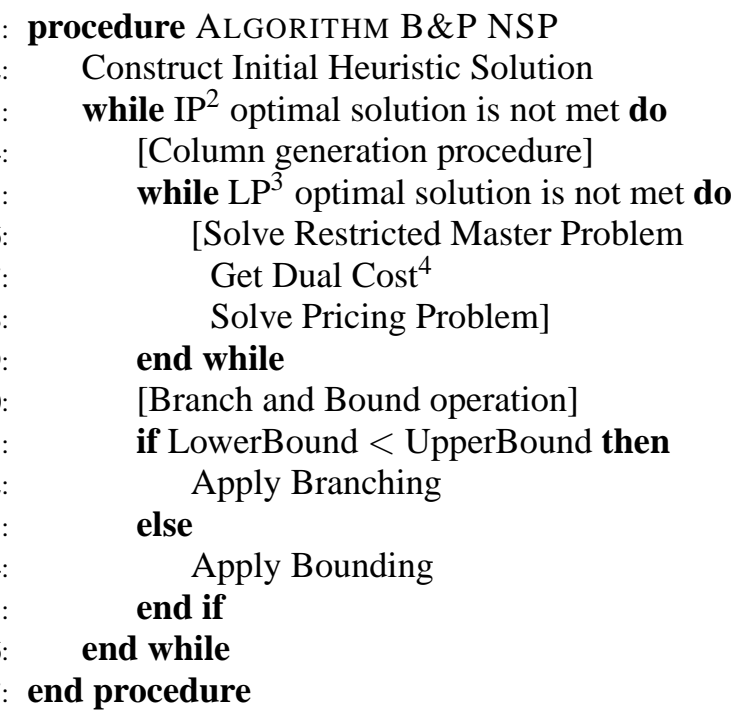

B\&P can be seen as a method of applying column generation to each subproblem in B\&B. The bounding and the branching operations of $\mathrm{B} \& \mathrm{~B}$ are performed based on the information derived by column generation. In B\&P, an exact real number solution of a relaxation problem in each subproblem can be derived by column generation, and an exact integer number solution (exact solution of original

2. Integer Programming

3. Linear Programming

4. The value of optimal dual variables in linear programming. problem) can be derived through the B\&B framework.

\subsection{Column generation}

In column generation, solutions are obtained by enumerating subsets of promising solutions and solving them as set-covering problems. Therefore, column generation can efficiently obtain a real valued optimal solution for problems with many variables and constraints.

In the column generation phase, a linear program called the master problem (MP) is optimized.

$$
\begin{gathered}
v(\mathrm{MP}):=\min \sum_{j \in J} c_{j} \lambda_{j} \\
\text { subject to } \sum_{j \in J} a_{j} \lambda_{j} \geq \mathbf{b} \\
\lambda_{j} \geq 0, j \in J
\end{gathered}
$$

In the above equation, $|J|=n$ is the number of variables and $m$ is the number of constraints. Since $|J|$ is usually huge, it is difficult to obtain an optimal solution on MP. Therefore, the restricted master problem (RMP), which contains only the subset $J^{\prime} \subseteq J$ of variables, is used instead of the MP.

In equation (9), $\lambda_{j}$ denotes the $j$ th column. $c_{j}$ and $a_{j}$ denote a cost and a variable for $\lambda_{j}$, respectively. $\lambda^{*}$ denotes optimal primal solutions for the RMP (If $\lambda_{j}=1$, then $\lambda_{j}$ is included in $\lambda^{*}$. Otherwise, if $\lambda_{j}=0$, then $\lambda_{j}$ is not included in $\lambda^{*}$.). $\pi^{*}$ denotes optimal dual solutions for the RMP. In most cases, RMP is solved by the simplex method. In the pricing step of the simplex method, we looked for a non-basis variable that is negative reduced cost and added it as a new basis variable. To accomplish this in column generation, the following pricing problem (or subproblem) PP must be solved.

$$
v(\mathrm{PP}):=\min \left\{c_{j}-\pi^{*} a_{j} \mid j \in J\right\}
$$

When $v(\mathrm{PP})<0$, the variable $\lambda_{j}$ and its coefficient column $\left(c_{j}, a_{j}\right)$ corresponding to $j$, which is to minimize $v(\mathrm{PP})$, is added to the RMP. This is optimally solved to obtain optimal dual variable values (dual costs), and the process iterates until no column provides further improvement. If no new columns with negative values can be found, the solution $\lambda^{*}$ to the restricted master problem can be considered to be optimally solved and this solution is also considered to be the optimal solution of the master problem (9).

The flow of the column generation is as follows.

Step 0 Prepare an initial set of columns, and construct a restricted master problem (RMP) which is a set covering problem only using the initial set of columns.

Step 1 Construct a linear relaxation problem relaxing the integer constraint of RMP, and obtain the optimal primal solution $\lambda^{*}$ and optimal dual solution $\pi^{*}$ to the covering problem. 


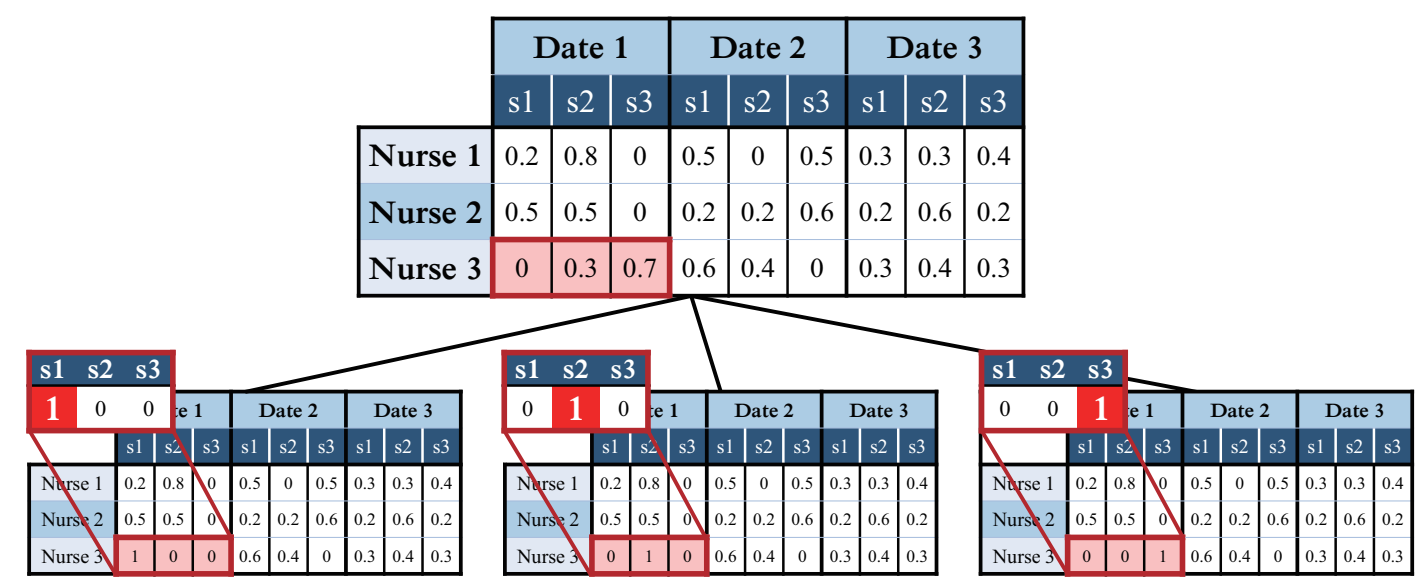

Fig. 2. Example graph for the part of branching tree

\begin{tabular}{|c|c|c|c|c|c|c|c|c|c|c|c|c|c|}
\hline \multirow{3}{*}{ Nurse $n$} & \multicolumn{3}{|c|}{ Date 1} & \multicolumn{3}{|c|}{ Date 2} & $\ldots$ & \multicolumn{3}{|c|}{ Date 27} & \multicolumn{3}{|c|}{ Date 28} \\
\hline & D & $\mathbf{N}$ & $\mathrm{O}$ & D & $\mathbf{N}$ & $\mathrm{O}$ & & D & $\mathrm{N}$ & O & D & $\mathbf{N}$ & O \\
\hline & 0 & 0 & 1 & 1 & 0 & 0 & $\cdots$ & 0 & 1 & 0 & 1 & 0 & 0 \\
\hline
\end{tabular}

Fig. 3. Example of column for one nurse

Step 2 Search the column in which the reduced cost $(\mathrm{rc})$ is negative $(\mathrm{rc}<0)$ by solving the pricing problem (PP) using the information of optimal dual solution $\pi^{*}$.

Step 2-1 If a column with negative reduced cost can be found, add that column to the RMP, and proceed to Step 1.

Step 2-2 If such a column is not found, terminate the process and assume that an optimal solution to the RMP has been obtained.

For the details of column generation, please refer to Desrosiers and Lübbecke's study[9].

It is possible to obtain an optimal solution and optimum value for the linear relaxation problem by using the column generation procedure described above. This optimum value is the lower bound value of the original problem in the $\mathrm{B} \& \mathrm{~B}$, and it can be used for the bounding operation. In addition, information for branching can be obtained by finding an optimal solution for each linear relaxation problem. And a real value of a variable in the optimal solution of linear relaxation problem changed to an integer value in the branching phase.

When applying the column generation to the NSP, each column represents the schedule of each nurse during a certain period, as in Fig. 3. The schedule in Fig. 3 represents the monthly schedule of one nurse, Off - Day ... - Night Day. Column generation can obtain a schedule like the one in Fig. 2 by repeating two processes. The first process is to generate a schedule (column) for each nurse. These schedules are stored in a column set called a column pool. The second process is to solve the RMP composed of a column pool as a set of cover problems. A schedule like the one in Fig. 2 is obtained by solving a set of cover problems. In most cases, tabu-search and dynamic programming are used to generate new columns.

\subsection{Branch and Bound}

An example of branching tree on NSP is shown in Fig. 2. In each node on the branching tree, a subproblem is created by fixing a part of shifts (s1, s2, s3) on a schedule of nurses. In B\&P, column generation is used to extract exact solutions to relaxation problems in subproblems.

After applying column generation, the values of lower bound (LB) and upper bound (UB) are compared in a branching strategy. LB is defined by an exact solution to the relaxation problem in each subproblem, and UB is calculated as the minimum objective value of the results obtained. Therefore, LB is a real number value, while UB is an integer number value. If $L B$ is smaller than the value of UB, the branching operation is performed. Otherwise, the bounding operation is called. When all nodes (subproblems) are solved, $\mathrm{B} \& \mathrm{~B}$ is terminated.

As mentioned above, $\mathrm{B} \& \mathrm{P}$ can derive a high-quality solution within feasible times by combining two mechanisms: column generation and $\mathrm{B} \& \mathrm{~B}$.

For a detailed explanation of how to implement $B \& P$ for NSP, please refer to Maenhout and Vanhoucke's study[2].

\section{Proposal Approach}

In this paper, we propose an effective new approach using $\mathrm{B} \& \mathrm{P}$ and heuristics to derive many high-quality schedules. Our approach first uses B\&P to produce an optimal schedule and then attempts to derive different schedules of almost the same quality with a heuristics algorithm. We use the evolutionary multi-objective optimization (EMO) algorithm as a heuristics algorithm because EMO is well suited to finding various solutions of roughly equal quality. Therefore, we call our approach B\&P-EMO in this paper. 


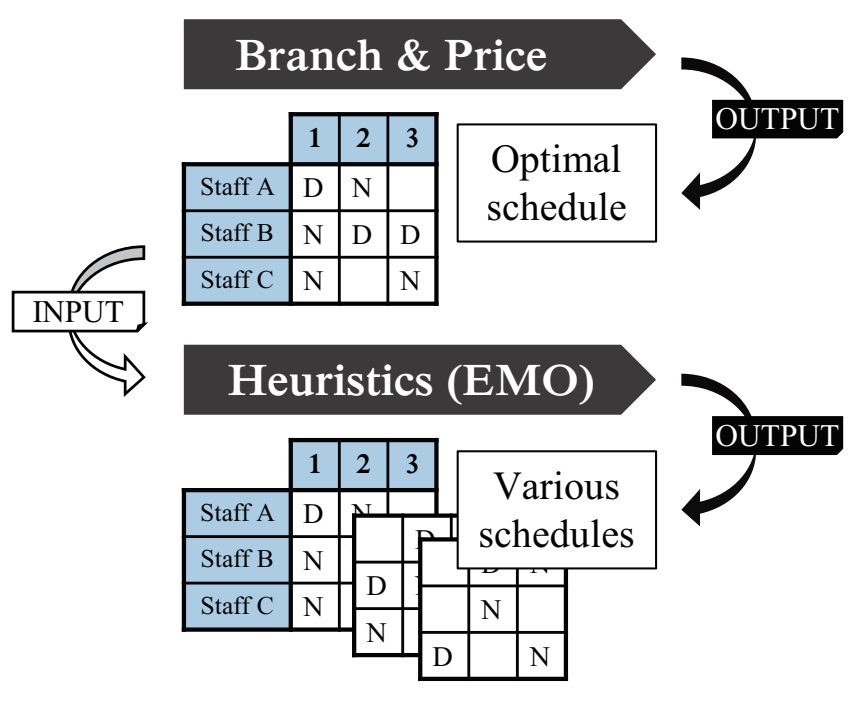

Fig. 4. Framework of B\&P-EMO

In this section, we first outline the B\&P-EMO and then describe the two mechanisms of B\&P-EMO in detail.

\subsection{Outline of B\&P-EMO}

B\&P-EMO first uses the B\&P algorithm to find an optimal schedule. However, as the B\&P algorithm cannot obtain different solutions directly, B\&P-EMO applies EMO algorithms to derive various schedules. The use of the optimal schedule obtained by $\mathrm{B} \& \mathrm{P}$ as an initial solution of EMO is an important aspect of B\&P-EMO. This makes it possible for EMO to search from the optimal schedule, finding solutions of nearly the same quality very efficiently.

The framework of B\&P-EMO is shown in Fig. 4. B\&P$\mathrm{EMO}$ first uses the $\mathrm{B} \& \mathrm{P}$ algorithm to attempt to find an optimal schedule, and then uses the EMO algorithm to attempt to derive more schedules of nearly the same quality.

The B\&P-EMO has two distinguishing points.

\section{- An efficient implementation of B\&P algorithm}

- A combination of B\&P and metaheuristics (EMO)

These two points are detailed below.

\subsection{Implementation of $B \& P$ algorithm}

In order to improve the search efficiency of B\&P, we implemented the following two ingenuities.

- A branching strategy considering the space efficiency and completeness of branch and bound

- The addition of an adjustment parameter for controlling the computing costs of dynamic programming to solve the pricing problem in column generation

These are explained in detail below.

\subsubsection{Branching Strategy Considering Space Efficiency and Completeness}

It is known that one of the most important keys to the $\mathrm{B} \& \mathrm{P}$ algorithm is how to implement an efficient tree search strategy. In most cases, a depth-first or breadth-first search is used in B\&B. Though depth-first has a low computing cost, the comprehensiveness of this strategy is low in the search space. On the other hand, though breadthfirst can search with high completeness, this strategy has a high computing cost. Therefore, we chose a search strategy that combines the space efficiency of the depth-first search with the completeness of the breadth-first search.

The concept of our search strategy is shown in Fig. 5. In this figure, the search point goes back to root node and tries to search another branching node when a bounding operation is performed. The purpose of this switching in our search strategy is to find an integer solution with good quality at an early stage of the search. The feature of our search strategy enables us to find high-quality integer solutions faster than is possible with a breadth-first search, and high-quality integer solutions are used in the bounding operation of $\mathrm{B} \& \mathrm{~B}$ as the upper bound.

\subsubsection{Adjustment Parameter for Controlling Computing Costs in Dynamic Programming}

In our $\mathrm{B} \& \mathrm{P}$, dynamic programming (DP) is used to create new columns in column generation. The DP in our research was implemented by refering to Burke and Curtois' mechanism[3]. In their approach, DP is treated as a resource-constrained shortest path problem. Fig. 6 shows an example graph in an instance with two shifts, Day and Night. This graph consists of an $n$th multistage network, and each stage corresponds to one workday of one nurse. In this figure, either Day, Night, or Off should be selected for each day. Their DP has two characteristics that make the search more efficient. The first is that path weights of network are represented by dual costs of RMP. The second is that infeasible routes are excluded in advance. These characteristics help to create new columns (new schedules) in PP more efficiently.

However, finding an exact solution in the pricing problem (PP) of column generation has large computing costs in this DP. This is because this PP of column generation needs to be re-optimized every time a new column is added, and this computation will be repeated until an optimal a solution of RMP cannot be improved.

Therefore, we add a threshold as an adjustment parameter when the PP of column generation is solved. The main benefit of this additional parameter is the PP can be solved roughly according to the situation in order to reduce computational costs. In our approach, the computational cost of solving PP will decrease when UB or LB is updated in a previous iteration. Conversely, when UB or LB is not updated in a previous iteration, this computational cost will not be saved.

In this study, we have tried to find a high-quality schedule within practicable times by using $\mathrm{B} \& \mathrm{P}$ having these two characteristics. 


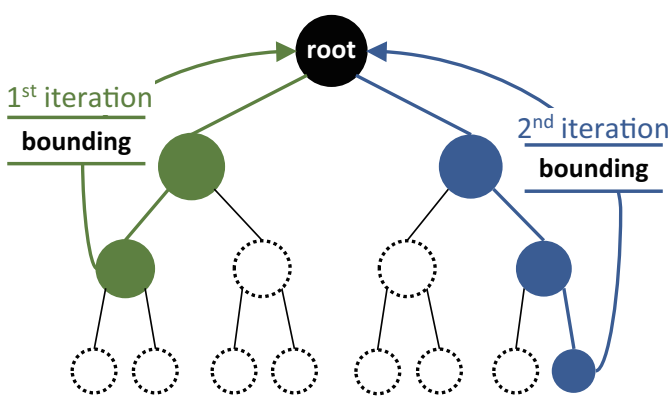

Fig. 5. Example graph of our searching strategy on B\&B

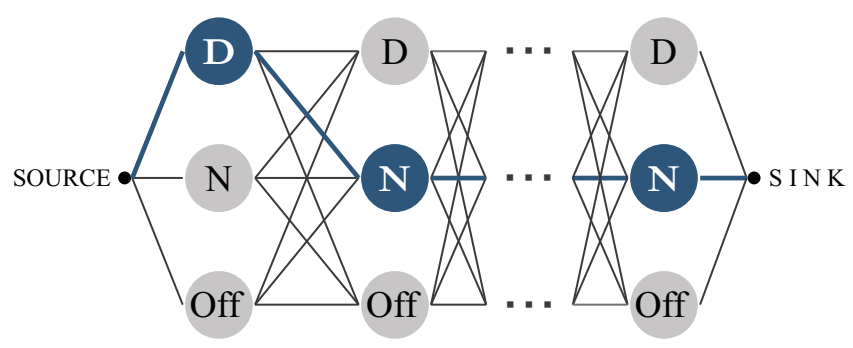

Fig. 6. Example graph for the shortest path problem

\subsection{A Combination of $B \& P$ and Metaheuristics (EMO)}

The B\&P algorithm can only derive one optimal solution, as is the case with other mathematical programming approaches. In this study, we have tried to combine B\&P and a heuristics algorithm to obtain many highly diverse solutions of almost the same quality as that of the optimal one. Therefore, we used the evolutionary multi-objective optimization (EMO) algorithm as a heuristic approach, because EMO based on multiple criteria is well suited to finding various non-dominated solutions.

The following is an outline of our EMO algorithm after $\mathrm{B} \& \mathrm{P}$ is applied.

\section{Step 0 Initialization}

Set solution derived by B\&P algorithm to initial solution of EMO. Copy this initial solution to population $P$.

\section{Step 1 Selection of individual for modification}

Select one individual $X \in P$ for modification. If the population was updated in the previous generation, select the best individual in the population. Otherwise, choose a non-dominated solution.

\section{Step 2 Update of solution}

Create new individuals by applying lengthwise perturbation to the selected individual $X$. Select best the solution from created individuals as $C_{\text {best }}$. Create new individual $Y$ by applying widthwise perturbation to $C_{\text {best }}$. If $f\left(C_{\text {best }}\right)>f(Y)$, then replace $C_{\text {best }}$ by $Y$ $\left(C_{\text {best }}=Y\right)$.

\section{Step 3 Updating of population}

Add $C_{\text {best }}$ to population $P$ according to updating criteria in section 4.3.3.

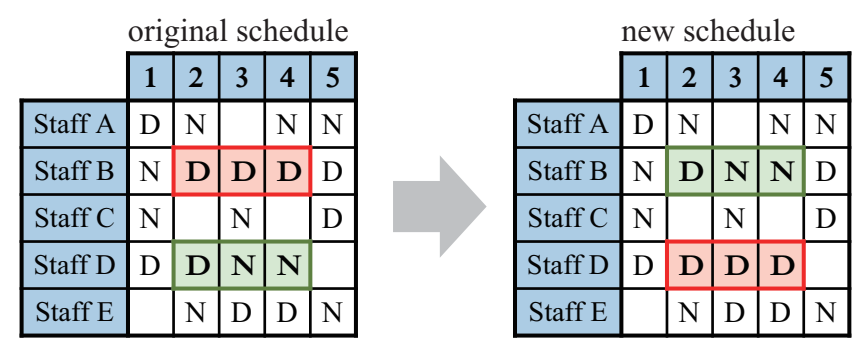

Fig. 7. Example of lengthwise perturbation

\section{Step 4 Termination criteria}

If the number of evaluations reaches a threshold, the search of EMO is finished. Otherwise, go back to Step 1.

The main aim of this EMO algorithm is to find various solutions of nearly the same quality as that of the optimal one. Therefore, our EMO algorithm was designed in consideration of the following two things.

- Modifying solutions very carefully so as not to violate satisfied constraints

- Using two criteria in the Step 3 when to update population: Pareto optimality and diversity of population.

Our EMO approach attempts to make new candidate solutions by modifying the (parent) solution very carefully in order to avoid lowering the quality of the solution. Specifically, our EMO approach uses two different exchanging operations, namely, lengthwise and widthwise perturbations, alternately and selects the best one after applying one operation but before applying the other operation in Step 2 above. Our EMO approach also uses diversity of population as a second criterion in order to obtain various solutions. Details of these features of our EMO approach follow.

\subsubsection{Operator of Lengthwise Perturbation}

An example of lengthwise perturbation is shown in Fig. 7. In this operation, new individual schedules are created by exchanging consecutive shift patterns between nurses on the same date. First, some nurses are selected and some new individual schedules are created by perturbing the shift patterns of the selected nurses. Then, the best individual schedule is selected from the ones created.

\subsubsection{Operator of Widthwise Perturbation}

An example of widthwise perturbation is shown in Fig. 8. In this operation, a new individual schedule is created by changing the shifts of a specific nurse to different dates. These changes are applied to a particular nurse so as not to violate the constraint related to the number of working days.

Changing the shifts of one nurse risks violating lengthwise constraints. Therefore, shift patterns are changed after investigating lengthwise constraints. 


\begin{tabular}{|c|c|c|c|c|c|c|c|c|c|c|c|}
\hline & \multicolumn{5}{|c|}{ original schedule } & & \multicolumn{5}{|c|}{ new schedule } \\
\hline & 1 & 2 & 3 & 4 & 5 & & 1 & 2 & 3 & 4 & 5 \\
\hline Staff A & $\mathrm{D}$ & $\mathrm{N}$ & & $\mathrm{N}$ & $\mathrm{N}$ & Staff A & D & $\mathrm{N}$ & & $\mathrm{N}$ & $\bar{N}$ \\
\hline Staff B & $\mathrm{N}$ & D & $\mathrm{D}$ & D & $\mathrm{D}$ & Staff B & $\mathrm{N}$ & $\mathrm{D}$ & $\mathrm{D}$ & D & $\mathrm{D}$ \\
\hline Staff C & $\mathrm{N}$ & & $\mathrm{N}$ & & $\mathrm{D}$ & Staff C & $\mathrm{N}$ & & $\mathrm{N}$ & & D \\
\hline Staff D & $\mathrm{D}$ & D & $\mathrm{N}$ & $\mathrm{N}$ & & Staff D & D & $\mathbf{N}$ & $\mathrm{N}$ & D & \\
\hline Staff E & & $\mathrm{N}$ & $\mathrm{D}$ & $\mathrm{D}$ & $\mathrm{N}$ & Staff E & & $\mathrm{N}$ & D & $\mathrm{D}$ & $\mathrm{N}$ \\
\hline
\end{tabular}

Fig. 8. Example of widthwise perturbation

\subsubsection{Updating of Population to Improve Optimality and Diversity of Solutions}

In updating a population, whether a new individual schedule that was created by applying each operator is to be added to the population is judged on two criteria: Pareto optimality and diversity of archive population. In EMO, (6) (Section 2.1) is used as the first objective function and the Hamming distance between a new individual schedule and the archive population is used as the second objective in order to derive various high-quality solutions.

Because two criteria based on optimality and diversity are used, solutions with not only high optimality but also high uniqueness can be found. Therefore, updating using these two criteria improves both the accuracy and diversity of the population.

\section{Numerical Experiments}

In this paper, we verified the effectiveness of $\mathrm{B} \& \mathrm{P}-$ EMO by applying our approach to several open benchmark instances. These instances were published on the website of Nottingham University (Employee Shift Scheduling Benchmark Data Sets)[4].

In this section, we present two different experiments.

1. An experiment to compare our approach with other existing approaches, such as E. K. Burke and T. Curtois's algorithms[3].

2. An experiment to show the diversity of the solutions derived by our approach.

In the first experiment, we tried to compare the quality as well as the computational cost of the solutions derived by our approach to those derived by other approaches.

In the second experiment, we tried to ascertain whether B\&P-EMO could derive many different schedules having almost the same quality as that of the optimal one. The features of the benchmark test instances are shown in Table 1. Each numerical experiment is described below.

\section{1. [experiment 1] Comparison of Search Abilities of our B\&P Algorithm and Other Existing Al- gorithms}

Table 2 presents the evaluation values of derived best solutions (UB) and computing times (t) (sec) used in our
$\mathrm{B} \& \mathrm{P}$ and Burke's B\&P[3]. It also presents evaluation values of solutions at the time of $600(\mathrm{sec})$.

Table 2 indicates that our B\&P algorithm was able to derive schedules equal to or greater than those derived by Burke's B\&P in all instances. In addition, it is clearly evident that our B\&P algorithm was able to derive optimal solutions in a very short time in small-scale instances, such as Millar-2Shift-DATA1 and LLR. Furthermore, in medium-scale instances such as Valouxis-1 and BCDTSep, our B\&P algorithm derived optimal solutions faster than did Burke's algorithm.

Comparing evaluation values of solutions at $600(\mathrm{sec})$ indicates that our algorithm derived higher-quality solutions at an early stage.

On the other hand, while our algorithm needed more computational time than Burke's algorithm did in the instance of Azaiez, it derived optimal schedules faster in other instances.

These results confirm that our algorithm can derive optimal solutions within realistic computational times on all scales.

\section{2. [experiment 2] Evaluation Related to Diver- sity of Solutions Derived by our Metaheuris- tics (EMO) Algorithm}

In this experiment, the size of the population was 32 , the maximum number of evaluations was 1 million, and the number of trials was 5 .

Table 3 shows the number of schedules derived by our EMO algorithm. These schedules consist of different shift patterns and are almost equal in quality to the optimal schedule derived by our B\&P algorithm. Table 3 indicates the minimum, average, and maximum values of the variances of these schedules in all trials.

In the LLR instance, our EMO algorithm successfully generated 20,000 schedules with different shift patterns from one optimal schedule. On the other hand, in the BCDT-Sep instance, our EMO algorithm was only able to derive 450 different schedules. It is interesting to note that the search space of LLR is smaller than that of BCDTSep.

These results seem to indicate that it is also difficult for our EMO algorithm to find different schedules in instances in which the B\&P algorithm has difficulty finding an optimal solution.

However, since our EMO algorithm derived at least 400 or more multiple schedules in all instances, we conclude that our EMO algorithm successfully found many different schedules of almost the same quality as that of the optimal one in all instances.

Moreover, the results in Fig. 9 to Fig. 13 show the Hamming distances of the solutions derived from the initial solution in all instances. For example, the bottom of Fig. 9 indicates that our EMO algorithm derived 58.2 schedules having small (from 1 to 10) Hamming distance values from the optimal solution derived by $\mathrm{B} \& \mathrm{P}$

Fig. 9 and Fig. 10 show the results of Millar-2ShiftDATA1 and LLR. The theoretical upper limits of the 
Table 1. Benchmark instances

\begin{tabular}{l|rrrrrl}
\hline Instance & staff & shift types & Length(days) & skill types & Best known & Reference \\
\hline Millar-2Shift-DATA1 & 8 & 2 & 14 & 1 & 0 & Ikegami and Niwa[10] \\
LLR & 27 & 3 & 7 & 1 & 301 & Li et al.[11] \\
Azaiez & 13 & 2 & 28 & 2 & 0 & Azaiez \& Al sharif[12] \\
Valouxis-1 & 16 & 3 & 28 & 1 & 20 & Valouxis and Housos[13] \\
BCDT-Sep & 20 & 4 & 30 & 1 & 100 & Bellanti et al.[14] \\
\hline \hline
\end{tabular}

Table 2. Results for proposed B\&P and Burke B\&P on benchmark instances

\begin{tabular}{l|rrrr|rrrc}
\hline Instance & \multicolumn{2}{|c}{ Our B\&P } & \multicolumn{2}{c|}{ Burke B\&P } & \multicolumn{2}{c}{ Our B\&P } & \multicolumn{2}{c}{ Burke B\&P } \\
& $\mathrm{UB}$ & \multicolumn{1}{c}{$\mathrm{t}(\mathrm{s})$} & $\mathrm{UB}$ & $\mathrm{t}(\mathrm{s})$ & $\mathrm{UB}$ & $\mathrm{t}(\mathrm{s})$ & $\mathrm{UB}$ & $\mathrm{t}(\mathrm{s})$ \\
\hline Millar-2Shift-DATA1 & 0 & 2.1 & 0 & $<0.1$ & 0 & 600.0 & 0 & 600.0 \\
LLR & 301 & 2.3 & 301 & 0.8 & 301 & 600.0 & 301 & 600.0 \\
Azaiez & 0 & 56.6 & 0 & 0.3 & 0 & 600.0 & 0 & 600.0 \\
Valouxis-1 & 20 & 73.0 & 80 & 909.6 & 20 & 600.0 & 160 & 600.0 \\
BCDT-Sep & 100 & 1547.9 & 100 & 6239.5 & 130 & 600.0 & 330 & 600.0 \\
\hline \hline
\end{tabular}

Table 3. The diversity of solutions derived by our B\&PEMO algorithm

\begin{tabular}{l|rrr}
\hline Instance & \multicolumn{3}{|c}{ Diversity of solutions } \\
& \multicolumn{1}{|c}{ min } & \multicolumn{1}{c}{ ave } & \multicolumn{1}{c}{$\max$} \\
\hline Millar-2Shift-DATA1 & 2233 & 2359.6 & 2575 \\
LLR & 19190 & 19827.6 & 20331 \\
Azaiez & 3673 & 3910.4 & 4207 \\
Valouxis-1 & 3613 & 4255.8 & 4526 \\
BCDT-Sep & 408 & 451.0 & 500 \\
\hline \hline
\end{tabular}

Hamming distance in these instances are 112 for the Millar-2Shift-DATA1 and 189 for the LLR. In these instances, our EMO algorithm derived schedules having one-third and more Hamming distance from the theoretical upper limit.

Fig. 11 and Fig. 12 show the Azaiez and Valouxis-1 results. Although there are no large differences between the theoretical upper limits in these instances, the maximum Hamming distances of the schedules derived by our EMO algorithm are completely different. In the case of Azaiez, the maximum Hamming distance was over 180, but this value was only over 60 for Valouxis-1. This result indicates that the performance of our EMO algorithm could depend not only on the scale of the instance but also on the other instances' settings, including constraints.

Fig. 13 presents the results of BCDT-Sep. Although BCDT-Sep has a higher theoretical upper limit of Hamming distance, our EMO algorithm was only able to derive schedules having 21 to 30 Hamming distance. However, over 250 schedules with 11 to 20 Hamming distance were derived, and we believe this number of schedules to be sufficient.

We also tried to analyze the contents of the derived schedules. Fig. 14 and Fig. 15 present the schedules of

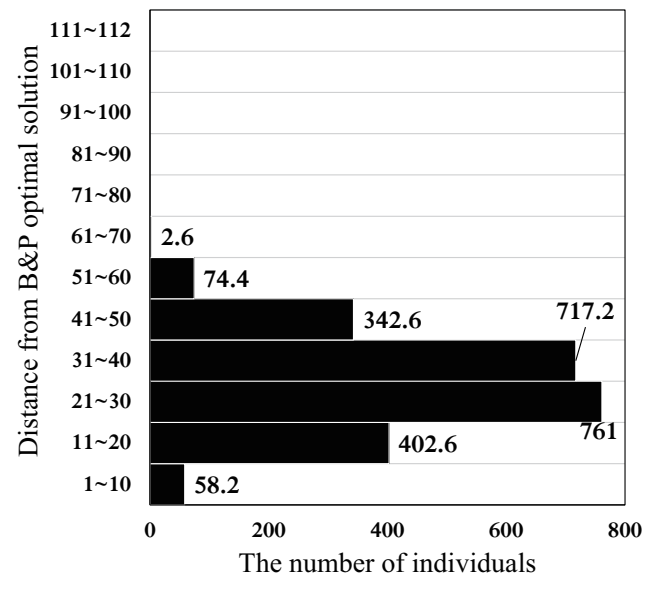

Fig. 9. Millar-2Shift-DATA1 results

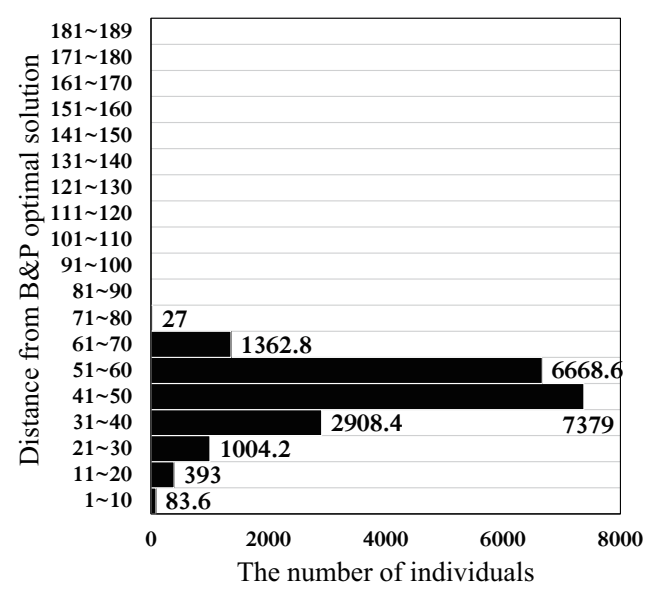

Fig. 10. LLR results 


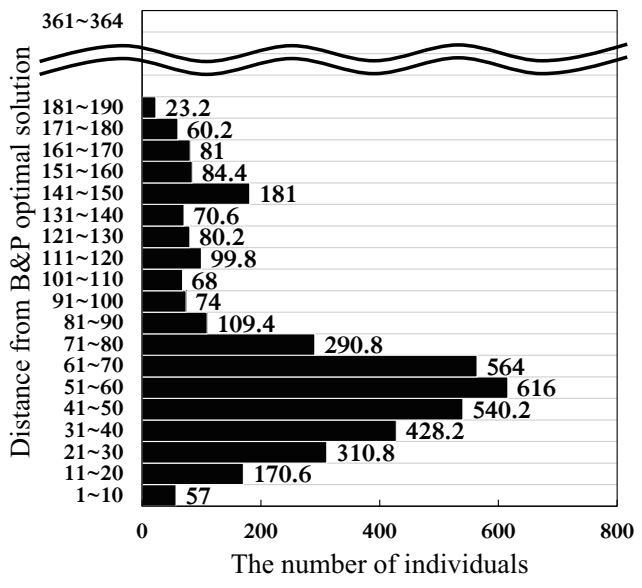

Fig. 11. Azaiez results

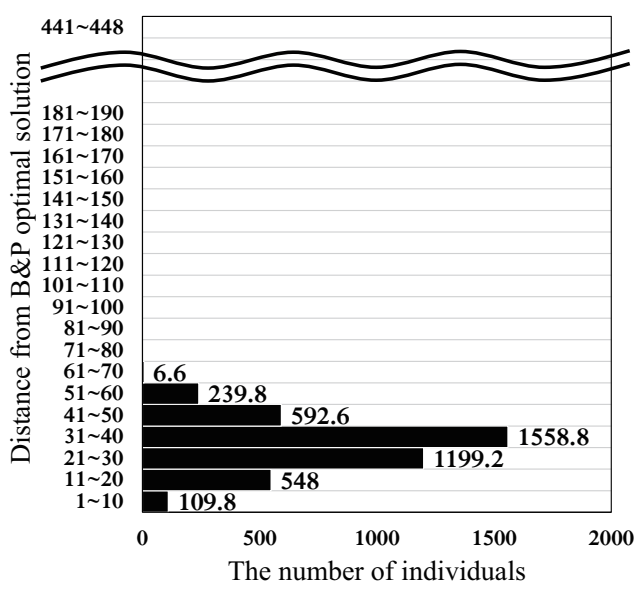

Fig. 12. Valouxis-1 results

Azaiez and Valouxis-1. In these figures, the upper schedule is the initial schedule derived by our B\&P algorithm, and the lower one is a schedule with maximum Hamming distance derived by our EMO algorithm. Red backgrounds in these schedules indicate shifts changed from those in the initial schedule.

These results show that most parts of the initial schedule were changed; only the schedule of Valouxis-1 remained largely unchanged. This tendency corresponded to that of the results of Hamming distances (Fig. 11 and Fig. 12).

These results indicate that the performance of our EMO algorithm depends on the characteristics of the instances, but the EMO algorithm successfully derived highly diverse schedules with in most instances.

\section{Conclusion}

In this paper, we proposed B\&P-EMO, a new approach to solving the nurse scheduling problem. B\&P-EMO is an algorithm that combines branch \& price with metaheuris-

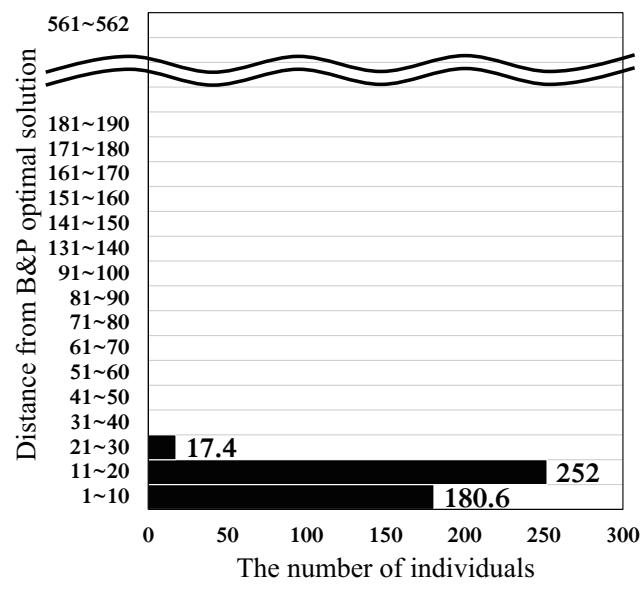

Fig. 13. BCDT-Sep results

tics (EMO algorithm). The main feature of B\&P-EMO is that it can derive many different schedules of almost the same quality as that of the optimal schedule within a practicable computational time.

The important points of $\mathrm{B} \& \mathrm{P}-\mathrm{EMO}$ are the following two mechanisms.

B\&P algorithm In the branch and bound method, we adopted a search strategy considering convergence and completeness, and we implemented a dynamic adjustment mechanism to save computational cost in the dynamic programming of the column generation method.

EMO algorithm The EMO algorithm created new schedules by replacing shifts in the lengthwise and widthwise directions of the schedule. In addition, by considering the criterion of diversity in updating the population, the EMO algorithm can improve the diversity of population.

We also investigated the effectiveness of our B\&PEMO by applying B\&P-EMO to several benchmark test instances published on Nottingham University's website[4]. Through these numerical experiments, we succeeded in obtaining the following results, confirming the effectiveness of our B\&P-EMO.

- Optimal schedules were derived within realistic times in all instances.

- Many different schedules of almost the same quality as that of an optimum schedule were derived.

In future works, we will present the characteristics of the derived schedules automatically by analyzing the relationships between the schedules.

\section{Acknowledgements}

This work was supported by JSPS KAKENHI Grants Number JP17K00331, JP17K01262 and JP16K00312. This was also partially supported by the "Joint Usage/Research Center for Interdisciplinary Large-scale Information Infrastructures " of Japan. 
Initial schedule

The part of difference shift

\begin{tabular}{|c|c|c|c|c|c|c|c|c|c|c|c|c|c|c|c|c|c|c|c|c|c|c|c|c|c|c|c|c|}
\hline & & & & 1 & & & & & & & 2 & & & & & & & 3 & & & & & & & 4 & & & \\
\hline & 2 & 3 & 4 & 5 & 6 & 7 & 8 & 9 & 10 & 11 & 12 & 13 & 14 & 15 & 16 & 17 & 18 & 19 & 20 & 21 & 22 & 23 & 24 & 25 & 26 & 27 & 28 & 29 \\
\hline & $M$ & $T$ & W & $\mathrm{T}$ & $\mathrm{F}$ & $S$ & $S$ & M & $\mathrm{T}$ & W & $\mathrm{T}$ & $\mathrm{F}$ & 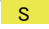 & $s$ & $M$ & $\mathrm{~T}$ & W & $\mathrm{T}$ & $\mathrm{F}$ & $s$ & $S$ & M & $\mathrm{T}$ & W & $\mathrm{T}$ & $\mathrm{F}$ & $S$ & $S$ \\
\hline G.M & D & & & $\mathrm{N}$ & $\mathrm{N}$ & $\mathrm{N}$ & $\mathrm{N}$ & & & D & D & & & & $\mathrm{N}$ & $N$ & $\mathrm{~N}$ & & & & D & & D & & & & & \\
\hline E.H & D & D & & & D & D & D & D & & & & & $N$ & $\mathrm{~N}$ & $\mathrm{~N}$ & & & D & D & & & $\mathrm{N}$ & $\mathrm{N}$ & $N$ & $N$ & & & \\
\hline M.B & $\mathrm{N}$ & $\mathrm{N}$ & $\mathrm{N}$ & $\mathrm{N}$ & & & $\mathrm{N}$ & $\mathrm{N}$ & $\mathrm{N}$ & & & D & D & & & & & $D$ & D & D & & & & D & D & & & D \\
\hline M.R & D & D & D & D & & & & L & $\mathrm{N}$ & $\mathrm{N}$ & $\mathrm{N}$ & $\mathrm{N}$ & & & & D & D & & 1 & $\mathrm{~N}$ & $\mathrm{~N}$ & $\mathrm{~N}$ & & & & & D & D \\
\hline D.M & $\mathrm{N}$ & $\mathrm{N}$ & $N$ & $\mathrm{~N}$ & & & D & D & D & & & & D & D & D & & & $\mathrm{N}$ & $N$ & $\mathrm{~N}$ & & & D & D & & & & \\
\hline V.A & $\mathrm{N}$ & $\mathrm{N}$ & & & $N$ & $\mathrm{~N}$ & & & D & D & D & D & & & & & & & $N$ & $\mathrm{~N}$ & $\mathrm{~N}$ & & & D & D & D & D & \\
\hline E.B & D & D & & & $\mathrm{N}$ & $\mathrm{N}$ & $\mathrm{N}$ & $\mathrm{N}$ & & & D & D & D & D & & & $\mathrm{N}$ & $\mathrm{N}$ & $\mathrm{N}$ & & & D & D & & & & & \\
\hline M.D & D & & & D & D & D & & & & $\mathrm{N}$ & $\mathrm{N}$ & $\mathrm{N}$ & $N$ & & & $\mathrm{~N}$ & $N$ & $N$ & & & & & D & D & D & D & & \\
\hline N.T & D & D & & & D & D & D & D & & & & & & $N$ & $\mathrm{~N}$ & $\mathrm{~N}$ & & & D & D & & & $\mathrm{N}$ & $N$ & $\mathrm{~N}$ & $\mathrm{~N}$ & & \\
\hline R.S & $\mathrm{N}$ & $\mathrm{N}$ & $\mathrm{N}$ & $\mathrm{N}$ & & & D & D & D & D & & 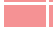 & & & & & D & D & & & D & D & & & & $N$ & $\mathrm{~N}$ & $\mathrm{~N}$ \\
\hline C.C & D & D & D & D & & & D & D & D & & & $\mathrm{N}$ & $\mathrm{N}$ & $N$ & $N$ & & & & & & & $\mathrm{~N}$ & $\mathrm{~N}$ & $\mathrm{~N}$ & & & & D \\
\hline R.C & D & D & D & D & & & & & $\mathrm{N}$ & $\mathrm{N}$ & $\mathrm{N}$ & & & D & D & D & D & & & & $\mathrm{N}$ & N & & & & & $\mathrm{N}$ & $\mathrm{N}$ \\
\hline M.N & $\mathrm{D}$ & $\mathrm{D}$ & D & D & & & & $\mathrm{N}$ & $\mathrm{N}$ & $\mathrm{N}$ & & & & & D & $D$ & & & & D & & & & & $\mathrm{N}$ & & $\mathrm{N}$ & $\mathrm{N}$ \\
\hline
\end{tabular}

Most distance schedule $($ distance $=185)$

\begin{tabular}{|c|c|c|c|c|c|c|c|c|c|c|c|c|c|c|c|c|c|c|c|c|c|c|c|c|c|c|c|c|}
\hline & \multicolumn{7}{|c|}{1} & \multicolumn{7}{|c|}{2} & \multicolumn{7}{|c|}{3} & \multicolumn{7}{|c|}{4} \\
\hline & 2 & 3 & 4 & 5 & 6 & 7 & 8 & 9 & 10 & 11 & 12 & 13 & 14 & 15 & 16 & 17 & 18 & 19 & 20 & 21 & 22 & 23 & 24 & 25 & 26 & 27 & 28 & 29 \\
\hline & $M$ & $\mathrm{~T}$ & W & $\mathrm{T}$ & $\mathrm{F}$ & S & S & M & $\mathrm{T}$ & W & $\mathrm{T}$ & $\mathrm{F}$ & S & $\mathrm{s}$ & $M$ & $\mathrm{~T}$ & W & $\mathrm{T}$ & $\mathrm{F}$ & S & $S$ & $M$ & $\mathrm{~T}$ & W & $\mathrm{T}$ & $\mathrm{F}$ & $S$ & $S$ \\
\hline G.M & $\mathrm{N}$ & & & $\mathrm{N}$ & $\mathrm{N}$ & $\mathrm{N}$ & $\mathrm{N}$ & & & D & D & & & & $\mathrm{N}$ & $N$ & & & D & $D$ & D & & & D & D & D & & \\
\hline E.H & D & & & D & D & D & D & & & & $\mathrm{N}$ & $\mathrm{N}$ & $\mathrm{N}$ & $\mathrm{N}$ & & & D & D & D & & & & & $N$ & $\mathrm{~N}$ & $N$ & & \\
\hline M.B & $N$ & $\mathrm{~N}$ & & & $\mathrm{~N}$ & $\mathrm{~N}$ & $\mathrm{~N}$ & $\mathrm{~N}$ & & & D & D & & & D & D & D & $\bar{D}$ & & & D & D & & & & & & $\mathrm{N}$ \\
\hline M.R & & D & D & D & D & & & $\mathrm{N}$ & $\mathrm{N}$ & $\mathrm{N}$ & & & D & D & D & D & & & & $N$ & $\mathrm{~N}$ & $\mathrm{~N}$ & $\mathrm{~N}$ & & & & & \\
\hline D.M & & & $\mathrm{N}$ & $\mathrm{N}$ & $\mathrm{N}$ & & & D & D & & & D & D & & & $\mathrm{N}$ & $\mathrm{N}$ & $\mathrm{N}$ & $\mathrm{N}$ & & & D & D & & & & D & D \\
\hline V.A & D & D & & & $\mathrm{N}$ & $\mathrm{N}$ & $\mathrm{N}$ & & L & D & D & & & & & & $\mathrm{N}$ & $\mathrm{N}$ & $\mathrm{N}$ & $\mathrm{N}$ & & & D & D & D & & & D \\
\hline $\mathrm{E} . \mathrm{B}$ & & $\mathrm{N}$ & $\mathrm{N}$ & & t. & & & $\mathrm{N}$ & $\mathrm{N}$ & $\mathrm{N}$ & & & D & D & D & D & & & $\mathrm{N}$ & $\mathrm{N}$ & & & & $\mathrm{D}$ & D & D & D & \\
\hline M.D & & D & D & & & D & D & & & $\mathrm{N}$ & $\mathrm{N}$ & $\mathrm{N}$ & $\mathrm{N}$ & & & $\mathrm{N}$ & $N$ & $N$ & & & D & D & D & D & & & & \\
\hline N.T & & & D & D & & & & D & D & & & $\mathrm{N}$ & $\mathrm{N}$ & $\mathrm{N}$ & $\mathrm{N}$ & & & D & D & D & & & $N$ & $\mathrm{~N}$ & $\mathrm{~N}$ & & & D \\
\hline R.S & $\mathrm{N}$ & & & N & $\mathrm{N}$ & & & $\bar{D}$ & D & & & D & D & D & D & & & & D & D & & & & $\mathrm{N}$ & $\mathrm{N}$ & $N$ & $\mathrm{~N}$ & \\
\hline C.C & D & & & D & D & D & & & & D & D & & & $\mathrm{N}$ & $\mathrm{N}$ & $\mathrm{N}$ & $\mathrm{N}$ & & & & $\mathrm{N}$ & $\mathrm{N}$ & $\mathrm{N}$ & & & D & D & \\
\hline R.C & D & D & & & D & D & & & $\mathrm{N}$ & $\mathrm{N}$ & & & & & D & D & D & D & & & $\mathrm{N}$ & $\mathrm{N}$ & & & & $\mathrm{N}$ & & $\mathrm{N}$ \\
\hline M.N & $\mathrm{N}$ & $N$ & $\mathrm{~N}$ & & & $\mathrm{D}$ & D & D & & & $\mathrm{N}$ & $\mathrm{N}$ & & & & & $D$ & & & & & & & & & & & $\mathrm{~N}$ \\
\hline
\end{tabular}

Fig. 14. The schedule of Azaiez derived by heuristic (EMO) algorithm

Initial schedule

The part of difference shift

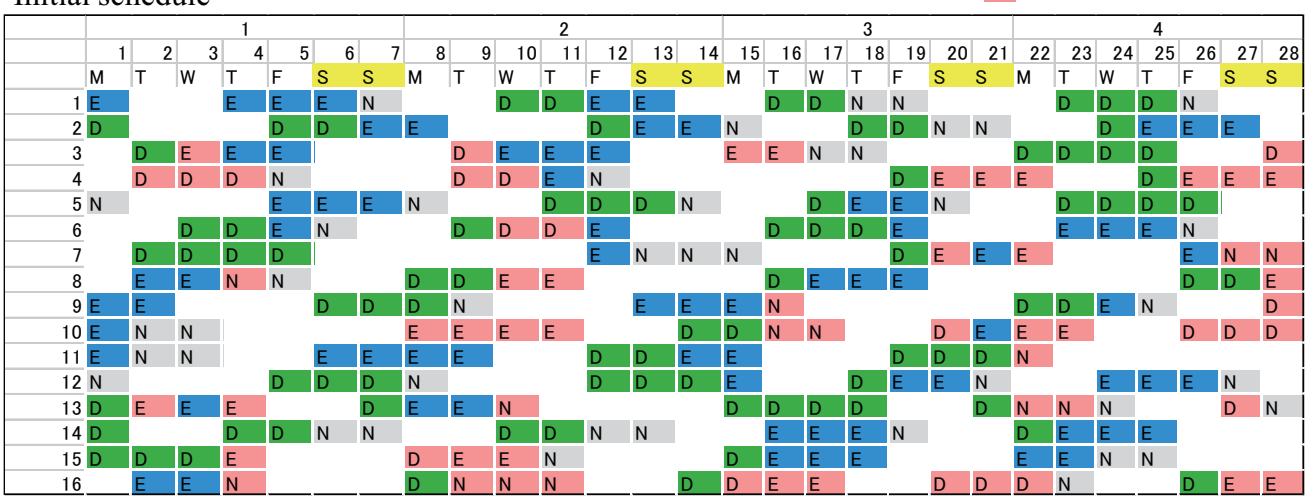

Most distance schedule $($ distance $=63)$

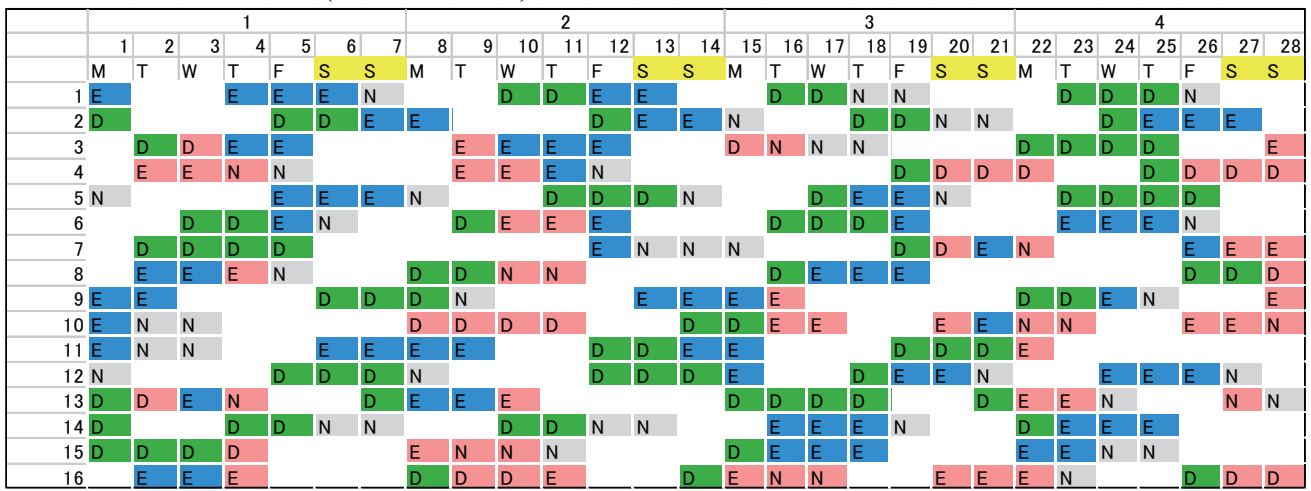

Fig. 15. The schedule of Valouxis-1 derived by heuristic (EMO) algorithm 


\section{References:}

[1] A. Ikegami, "A Model for the Nurse Scheduling Problem", IPSJ SIG Notes, 96(10):1-6, jan 1996.

[2] B. Maenhout and M. Vanhoucke, "Branching strategies in a branchand-price approach for a multiple objective nurse scheduling problem", Journal of Scheduling, 13(1):77-93, 2010.

[3] E. K. Burke and T. Curtois, "New approaches to nurse rostering benchmark instances", European Journal of Operational Research, 237(1):71 - 81, 2014.

[4] T. Curtois. "Employee Shift Scheduling Benchmark Data Sets". Available from: http://www.cs.nott.ac.uk/psztc/NRP/.

[5] A. A. Musa and U. Saxena, "Scheduling Nurses Using GoalProgramming Techniques", IIE Transactions, 16(3):216-221, 1984

[6] I. Ozkarahan, "The zero-one goal programming model of a flexible nurse scheduling support system", JIn Proceedings of international industrial engineering conference, pp. 436-441, 1989.

[7] A. Ikegami and A. Niwa, "A subproblem-centric model and approach to the nurse scheduling problem", Mathematical Programming, 97(3):517-541, Aug 2003.

[8] E. K. Burke, T. Curtois, G. Post, R. Qu, and B. Veltman, "A hybrid heuristic ordering and variable neighbourhood search for the nurse rostering problem", European Journal of Operational Research, 188(2):330 - 341, 2008.

[9] J. Desrosiers and M. E. Lübbecke, "A Primer in Column Generation”, pp. 1-32, Springer US, Boston, MA, 2005.

[10] A. Ikegami and A. Niwa. "A Subproblem-centric Model and Approach to the Nurse Scheduling Problem.", 2003.

[11] H. Li, A. Lim, and B. Rodrigues, "A Hybrid AI Approach for Nurse Rostering Problem", In Proceedings of the 2003 ACM Symposium on Applied Computing, SAC '03, pp. 730-735, New York, NY, USA, 2003, ACM

[12] M. N. Azaiez and S. S. Al Sharif, "A 0-1 Goal Programming Model for Nurse Scheduling”, Comput. Oper. Res., 32(3):491-507, March 2005.

[13] C. Valouxis and E. Housos, "Hybrid optimization techniques for the workshift and rest assignment of nursing personnel", Artificial Intelligence in Medicine, 20(2):155 - 175, 2000. Planning and Scheduling in the Hospital.

[14] F. Bellanti, G. Carello, F. D. Croce, and R. Tadei, "A greedy-based neighborhood search approach to a nurse rostering problem", European Journal of Operational Research, 153(1):28 - 40, 2004. Timetabling and Rostering.

Name:

Junya INAFUNE

\section{Affiliation:}

Muroran Institute of Technology

Address:

Address of Your Institute

Brief Biographical History:

Your History

Main Works:

- Your Works

Membership in Academic Societies:

- Your Learned Societies 